\title{
Contre l'âgisme et pour les super-vieux
}

Quand promouvoir prévention et soins chez les plus vieux passe par le changement des représentations sociales

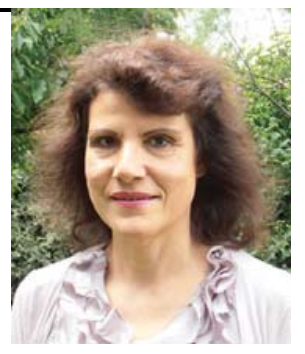

\author{
Against Agism and pro Super-Elderly \\ When promoting prevention, care and cure in the Elderly means \\ changing social stereotypes
}

\author{
M.-F. Bacqué \\ (C) Springer-Verlag France 2012
}

Un octogénaire plantait.

"Passe encor de bâtir ; mais planter à cet âge!"

Disaient trois jouvenceaux, enfants du voisinage ;

Assurément il radotait.

"Car, au nom des dieux, je vous prie,

Quel fruit de ce labeur pouvez-vous recueillir?

Autant qu'un patriarche il vous faudrait vieillir.

A quoi bon charger votre vie

Des soins d'un avenir qui n'est pas fait pour vous?»

Le vieillard et les trois jeunes hommes Jean de La Fontaine

\section{Il y avait le sexisme}

Depuis quelques années, on admet l'âgisme. L'âgisme est au vieillissement ce que le racisme et le sexisme sont aux différences physiques. L'âgisme est l'ensemble de toutes les discriminations liées à l'âge. Il peut donc aussi bien concerner les jeunes que les plus âgés (on ne parlera de jeunisme que lorsqu'il s'agit d'un engouement lié au jeune âge). Le concept, créé par le gérontologue Robert Butler en 1969, est aujourd'hui surtout employé pour dénommer la dévalorisation liée à l'âge des seniors [1,2]. En France, il est développé depuis les années 2008, et sans doute plus concrètement à partir du Québec qui effectue un travail militant en la matière (lire le rapport du Conseil des aînés

M.-F. Bacqué $(\bowtie)$

EA3071, Université de Strasbourg - Rédactrice en chef

de la revue Psycho-Oncologie

e-mail : mfbacque@club-internet.fr du Québec en 2010) et qui voit Martine Lagacé [8] publier le premier ouvrage en français sur l'âgisme en 2010.

Lodovico Baldocci le constate dans ce numéro, l'âgisme fait des ravages dans les sociétés sur le plan du travail, de la sexualité, de l'économie, de la formation des seniors. Mais sur le plan de la maladie-cancer et du soutien des aidants âgés, la situation est encore très empreinte de stéréotypes et de préjugés à l'encontre des « très grands vieux ».

\section{Âgisme et prévention du cancer du sein}

Prenons le cancer du sein. L'association AGE (voir la plateforme européenne des personnes âgées) demandait dès le 31 octobre 2008 que le programme de dépistage organisé du cancer du sein qui permet « de bénéficier, tous les deux ans, d'une mammographie prise en charge à $100 \%$ par l'Assurance Maladie sans avance de frais et dans des conditions contrôlées de qualité et de sécurité », soit proposé sans limite d'âge aux françaises. Jusqu'à présent, il concernait les femmes âgées de 50 à... 74 ans. Or, l'incidence des cancers du sein est à peu près équivalente, de 55 ans jusqu'à 85 ans. Pourquoi discriminer les femmes de plus de 74 ans ? Ne s'agit-il pas ici d'une « perte de chance » face à un cancer curable qui peut être particulièrement angoissant quand on est vieille et que les préjugés concernant la finitude de la beauté, la ménopause ou encore tout simplement la possibilité de se soigner, prévalent de manière larvée dans la société [10]?

Ainsi, Teuscher et Teuscher [12] montrent que les femmes âgées sont doublement victimes d'une dévalorisation du fait de leur âge, mais qui est encore augmentée du fait de leur sexe ! Les femmes, à âge égal, sont en effet généralement vues comme étant plus âgées que les hommes. D'autre part, en raison de l'âgisme, elles sont perçues plus négativement que leurs congénères masculins. Ceci se traduirait par 
une exposition des femmes plus longue et plus forte que les hommes, au phénomène de rejet des personnes âgées.

\section{L'âgisme chez les médecins et personnels de santé}

Mais pour les soignants et les médecins, les stéréotypes projetés sur la population des seniors ne sont-ils pas encore plus choquants?

Rappelons que chez nos voisins du Royaume-Uni, une enquête de l'association britannique Help the aged, menée auprès de deux cents médecins membres de la Société britannique de gériatrie, montre que $72 \%$ des médecins interrogés pensent que les personnes âgées bénéficient moins souvent de traitements neurochirurgicaux ou de chimiothérapies anti-cancéreuses. Ils sont $77 \%$ à se déclarer favorables à des lois contre la discrimination liée à l'âge dans l'accès aux prestations du service national de santé (NHS).

Dans ce numéro, Florence Barruel observe que de nombreuses pratiques soignantes françaises sont infiltrées par l'âgisme et se traduisent par :

- ne pas informer de la maladie, mentir ou au contraire brusquer le patient qui « en a vu d'autres »;

- ne pas soigner le patient comme un autre plus jeune, car il serait « trop fragile », destiné à mourir rapidement, voire même libéré plus vite du fardeau de la vie...

- ne pas prendre en compte les aidants trop âgés car ils ne comprennent jamais rien, ils sont dépassés, leur amour et leur tendresse pour le compagnon âgé sont suspects...

\section{Âgisme des psychologues et psychiatres}

Pour les psychologues et les psychiatres, les observations se corsent. En effet, s'intriquent plusieurs dimensions qui relèvent des médecins et psychothérapeutes, mais aussi des malades âgés eux-mêmes. Une certaine auto-dévalorisation conduit les malades âgés à minimiser leurs douleurs, leurs plaintes et parfois même leurs traitements. Selon Yves Libert et son équipe, dans ce numéro, ils sont relativement peu nombreux à demander une aide psychologique $(16 \%$ sur 330 patients inclus dans l'étude, ceux qui présentaient une détresse psychologique modérée à sévère n'étaient pas les plus nombreux à demander cette aide psychologique). La question de l'évaluation des capacités cognitives, mais surtout des capacités de jugement et de raisonnement est primordiale et rappelée dans les recommandations de la SFPO. Mais qu'en est-il des représentations sociales et psychologiques qui pèsent lourdement sur les très grands vieux et leur psychopathologie ? Selon l'Alliance for Aging Research (2003), les aînés sont moins susceptibles d'être testés, ils sont souvent exclus des essais cliniques pharmacologiques ou encore assimilés à une seule classe d'âge (les plus de 65 ou les plus de 75 ans) alors que des changements complets peuvent intervenir pendant ces dizaines d'années, en raison de l'usure biologique, de la perte du conjoint ou... de l'augmentation des stéréotypes. Saint-Jean et Somme notaient déjà en 1999 [11] que l'âgisme ne devrait pas être un élément régulateur des dépenses de santé, comme cela est toujours constaté en Europe, au Canada où les personnes âgées reçoivent souvent des traitements « édulcorés » par rapport aux jeunes adultes, ce que Florence Joly, avait constaté en appelant de ses vœux [7] la diffusion de la nouvelle discipline qu'est l'onco-gériatrie en 2006. Ainsi, Dozois, à la même époque [6], observait que la plupart des interventions en termes de politique de santé s'adressant aux aînés, visent le contrôle des symptômes et le traitement des maladies, tandis que la prévention et la promotion de la santé restent négligées. Attribuer des symptômes au processus normal du vieillissement plutôt qu'au développement d'une tumeur est banal dans le cas de la démence, mais c'est souvent la dépression qui fait le lit des absences d'investigation. Bizzini et Rapin [1] admettent que la dépression chez les aînés est encore trop souvent sous-estimée, sous-diagnostiquée et sous-traitée par les cliniciens. Seul, 10\% des personnes âgées présentant une dépression, recevrait des traitements adéquats. L'évaluation psychologique, voire l'évaluation de psycho-gériatrie plus poussée [5], doivent systématiquement être proposées aux patients âgés, comme nous le recommande la SFPO (vous trouverez dans l'article de Sarah Dauchy et al. toutes les échelles et questionnaires nécessaires pour les pratiquer).

Et pour les préjugés des psychothérapeutes, nous sommes également servis ! Lee, Volans et Gregory [9] se sont intéressés aux attitudes des psychothérapeutes vis-à-vis de la clientèle âgée. Le préjugé habituel (y compris chez Freud) voulait que les personnes âgées soient plus résistantes aux effets de la psychothérapie. Les psychologues et psychiatres se sentent donc moins enclins à la leur proposer. Ils privilégieraient même la thérapie de soutien, dans une visée à court terme impliquant l'idée que les patients sont proches de la mort. Parfois même, ils refusent ce type de patients. Or, les conséquences d'un symptôme, voire d'un syndrome psychopathologique, surtout la dépression, sont considérables en termes de qualité de vie et peuvent mener au suicide sur le plan quantitatif.

\section{Quelles propositions pour lutter contre l'âgisme en psycho-oncologie ?}

Informer les médecins et les soignants nous a paru primordial car cette population spécifique doit être ciblée par les politiques de santé au plus vite pour limiter la perte de 
chance des patients âgés. J'insisterai même pour que l'âgisme ne se double pas de sexisme. Hélas tant que le nombre de femmes médecins, chef de service et chercheures n'augmentera pas, nous savons que les discriminations des femmes les laisseront loin derrière. Rappelons-nous que la question de l'impuissance masculine liée à certains traitements du cancer de la prostate est un sujet de recherche qui semble rencontrer beaucoup plus de succès que les difficultés liées à la ménopause induite par les thérapies anti-cancer chez les femmes.

Modifier les représentations de la société française est un cheval de bataille beaucoup plus délicat à enfourcher... Cependant, les images des " très grands vieux » pourraient être modifiées, par exemple pourquoi ne pas trouver une dénomination plus sympathique, plus dynamique pour les nouveaux vieux que nous serons dans vingt ans, dix ans, demain ?

\section{Des super-vieux...}

Ce terme pourrait être attribué à la génération prochaine de plus de 70 ans. En forme, mieux nourris que leurs parents, mieux à même d'avoir appliqué la prévention des maladies graves, ils pourraient disposer d'un revenu leur permettant de se cultiver, voyager, mais aussi et surtout échanger avec les autres générations. Trop souvent encore, cette nouvelle génération est représentée comme égoïstement immature, frondeuse et solitaire (n'est-elle pas adepte du téléphone portable, du golf et des croisières ?). Ces images toutes faites, ces préjugés collent à la peau des retraités (riches) parce que nous avons peur du vieillissement. La théorie de la gestion de la peur - ou de la terreur, selon Valerian Boudjemadi et Kamel Gana [4] - expliquerait en partie les préjugés de l'âgisme. La plupart d'entre nous chercherait en effet à se protéger de l'angoisse de mort, soit en déplaçant la peur de la mort sur celle du vieillissement, soit en projetant la menace sur les très grands vieux et en évitant la confrontation à la détérioration physique et cognitive redoutée par dessus tout. Le groupe ainsi perçu devient alors minoritaire et vulnérable, ce qui est justifié par les stéréotypes qui rationalisent ces idées inconscientes au départ.

\section{Une promotion de la prévention et du dépistage, spécifique à chaque génération}

L'épidémiologie est une discipline qui doit intégrer les représentations sociales. Les personnes retraitées sont très différentes en fonction de leur niveau socio-économique, mais surtout en fonction de leur âge. Ainsi, la catégorie des « plus de 65 ans » ne devrait plus exister mais se diviser en 65-75, $75-85,85-95,95$ et plus. Cette diversification limiterait les « blocs » de préjugés à l'encontre de ces personnes et permettrait enfin de favoriser l'écoute et la singularité. Geneviève Laroque, Présidente de la Fondation Nationale de Gérontologie a écrit un très beau texte à l'occasion de la Journée Européenne de la solidarité entre les générations (29 avril 2009). Elle insiste pour le partage des valeurs entre générations comme caution de notre vie démocratique et humaniste.

Seuls la solidarité et le partage permettront à chaque génération de se sentir dotée et de trouver sa place au cœur de la société. Rien de pire en effet que la mort sociale qui dénie à l'individu ses rôles. Parmi ces rôles, la mémoire, la sagesse de l'expérience ou des erreurs passées, l'anticipation de l'avenir sont les qualités subtiles du grand vieillissement.

Nous remercions Valérian Boudjemadi (LPC - Université de Strasbourg) pour sa relecture attentive et enrichissante.

\section{Références}

1. Bizzini L, Rapin CH (2007) L'âgisme : une forme de discrimination qui porte préjudice aux personnes âgées et prépare le terrain de la négligence et de la violence. Gérontologie et sociéte 123:263-278

2. Butler RN (1969) Age-Ism : Another form of bigotry. The Gerontologist 9(4):243

3. Butler RN (1978) Thoughts on aging. American Journal of Psychiatry $135: 14-16$

4. Boudjemadi V, Gana K (2009) L'âgisme : adaptation française d'une mesure et test d'un modèle structural des effets de l'empathie, l'orientation à la dominance sociale et le dogmatisme sur l'âgisme. Revue Canadienne du Vieillissement 28:371-389

5. Chaïbi P, Magné N, Breton S, et al (2011) Influence of geriatric consultation with comprehensive geriatric assessment on final therapeutic decision in elderly cancer patients. Crit Rev Oncol Hematol 79(3):302-7

6. Dozois E (2006) Ageism: A review of the litterature. Calgary Health Region, Healthy Aging Committee, 29 p.

7. Joly F (2006) L'âge est-il toujours un facteur limitant dans la prise en charge des patients atteints de cancer ? Rev Francoph Psycho-Oncologie 3:137-138

8. Lagacé M (sous la direction de) (2010) L'Âgisme. Comprendre et changer le regard social sur le vieillissement. Presses de l'Université Laval, Québec

9. Lee KM, Volans PJ, Gregory N (2003) « Attitudes towards psychotherapy with older people among trainee clinical psychologist », Aging \& Mental Health 7(2): 133-141

10. Pritchard KI (2007) Have we been guilty of ageism in the primary treatment of breast cancer? British Journal of Cancer 96:1011-1012

11. Saint-Jean O, Somme D (1999) Âge et restriction d'accès aux soins. Gérontologie et société 90:109-12

12. Teuscher U, Teuscher $\mathrm{C}$ (2006) Reconsidering the double standard of aging. Effects of gender and sexual orientation on facial attractiveness rating. Personality and Individual Differences 42:631-39 


\section{Quelques références internet}

AGE - la plateforme européenne des personnes âgées : www.age-platform.org

Avis sur l'âgisme envers les aînés - état de la situation. Conseil des aînés du Québec Mars 2010 : www.conseil-des-aines.qc.ca
Fédération Internationale des Associations de Personnes Âgées (FIAPA) : www.fiapa.org

Fédération Internationale sur le Vieillissement (FIV) : http://www.ifafiv.org

HelpAge International : http://www.helpage.org

Observatoire de l'âgisme (2008). Lettre à la HALDE : http://www. agisme.fr/spip.php?article9 